\title{
A Comparison between Standard and Crossfeed Monopulse Radars in Presence of Rough Sea Scattering and Ship Movements
}

\author{
Giorgio Giunta, ${ }^{1}$ Leonardo Lucci, ${ }^{2}$ Renzo Nesti, ${ }^{3}$ Giuseppe Pelosi, ${ }^{2}$ \\ Stefano Selleri, ${ }^{2}$ and Francesco Serrano ${ }^{1}$ \\ ${ }^{1}$ Antenna development \& EMC, Rheinmetall Italia SpA, Via Affile 102, 00131 Rome, Italy \\ ${ }^{2}$ Department of Electronics and Telecommunications, University of Florence, Via C. Lombroso 6/17, 50134 Florence, Italy \\ ${ }^{3}$ Astrophysical Observatory of Arcetri, National Institute for Astrophysics, Largo E. Fermi 5, 50125 Florence, Italy
}

Correspondence should be addressed to Leonardo Lucci, leonardo.lucci@unifi.it

Received 20 May 2010; Accepted 23 July 2010

Academic Editor: Tat Yeo

Copyright (c) 2010 Giorgio Giunta et al. This is an open access article distributed under the Creative Commons Attribution License, which permits unrestricted use, distribution, and reproduction in any medium, provided the original work is properly cited.

\begin{abstract}
Monopulse radars are widely used in tracking systems, due to their relative simplicity and theoretical precision, but the presence of multipath impairs the tracking capabilities of these radars, especially when multipath signals are strong, as in a naval environment. A special monopulse setup, the crossfeed, has been proposed in the past to provide an automatic cancellation from smooth sea multipath. In this contribution, the performances of such a system are analyzed in presence of rough sea scattering and compared with those of a standard monopulse setup. Particular attention is devoted to performance degradations due to possible phase errors in the passive network implementing the comparator and due to ship rolling and pitching. This latter requires a full 3D monopulse simulator for its correct evaluation.
\end{abstract}

\section{Introduction}

Accurate tracking of an incoming target is a fundamental issue in defense systems. The preferred technique is that of monopulse radars [1-3]. These radars are small enough to mechanically track, in real time, a fast moving target yet very accurate in their pointing. Such accuracy is obtained by exploiting the null, rather than the main beam, of an appropriate pattern, the null being usually much sharper than the maximum. Real-time tracking is achieved via a control system evaluating and minimizing the signal received through the pattern realizing the null.

It is easy to comprehend that such devices are very sensitive to multipath as the multipath rays, even if received from a direction very close to the direct ray, can produce relevant signals in the pattern realizing the null.

The multipath problem is indeed very critical over the sea surface and for low elevation angles. In [4], an overview of the theories for the interaction of electromagnetic and oceanic waves is reported, and in [5-11], the problem of multipath effects on a "standard" monopulse both over smooth and rough sea has been addressed. In the literature, solutions have been proposed exploiting multiple radars [12, 13] or frequency agility [14] to overcome the depointing due to multipath. Some authors have also proposed a crossfeed monopulse [14] which is able, theoretically, to perfectly cancel out the sea multipath, hence, allowing a perfect tracking.

The crossfeed monopulse has been analyzed in [15] only in presence of a smooth isoreflective sea. In this paper, the configuration is tested over a realistic rough sea. Furthermore, its real-world performances are evaluated by taking into account possible errors in the waveguide beamforming network backing the antenna.

It is also worth noticing that nearly all papers present simulations in a vertical plane containing both the antenna and the target. This is not enough if ship rolling and pitching are to be taken into account, hence, in this paper, a full 3D monopulse simulator will be outlined. Some very preliminary results have been presented $[16,17]$ for what concerns a crossfeed over a rough sea in the $2 \mathrm{D}$ case. This paper presents the 3D case comprehensive of ship roll and pitch movements. 


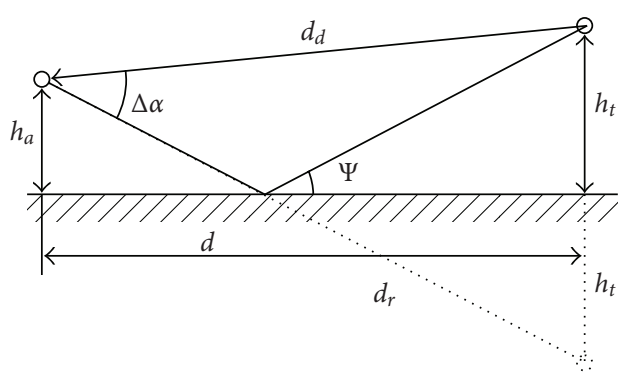

FIGURE 1: Multipath problem for an antenna (left) and a target (right) above a flat sea surface.

The paper is organized as follows. In Section 2, the basics of rough sea multipath will be recalled, while Section 3 will present the standard monopulse setup and show the crossfeed setup, which is more difficult to find in the literature. Section 4 will compare the performances of the two configurations for an ideal antenna in presence of rough sea. Sections 5 and 6 will present the effects of manufacturing tolerances and ship movements on tracking capabilities and some consideration over real antennas will be also presented. Finally, Section 7 will draw the conclusions.

\section{Rough Sea Multipath}

In a system comprising the radar, the target, and the sea surface (Figures 1 and 2), four possible paths are possible:

(i) antenna-target-antenna,

(ii) antenna-target-sea-antenna,

(iii) antenna-sea-target-antenna,

(iv) antenna-sea-target-sea-antenna.

In a low target configuration, all four paths are theoretically to be taken into account but, given the fact that the target is unknown, the way it is illuminated is irrelevant, hence, the problem can be reduced to a unitary source replacing the target and to the two paths leading from the target to the radar. Figure 1 shows indeed this case, for a flat earth, with just two rays, the directed and the reflected, traveling from the target ( $h_{t}$ meters above the sea) to the antenna ( $h_{a}$ meters above the sea). The mutual distance among the two is $d$. The reflected signal impinges on the sea surface with an angle $\psi$ from the grazing direction. The direct and reflected rays are $d_{d}$ and $d_{r}$ meters long, respectively, and exhibit a difference in their direction of arrival at the antenna level equal to $\Delta \alpha$ :

$$
\begin{gathered}
d_{d}=\sqrt{d^{2}+\left(h_{a}-h_{t}\right)^{2}}, \\
d_{r}=\sqrt{d^{2}+\left(h_{a}+h_{t}\right)^{2}}, \\
\Delta \alpha=\sin ^{-1}\left(\frac{h_{t}+h_{a}}{d_{r}}\right)+\sin ^{-1}\left(\frac{h_{t}-h_{a}}{d_{r}}\right), \\
\psi=\sin ^{-1}\left(\frac{h_{t}+h_{a}}{d_{r}}\right) .
\end{gathered}
$$

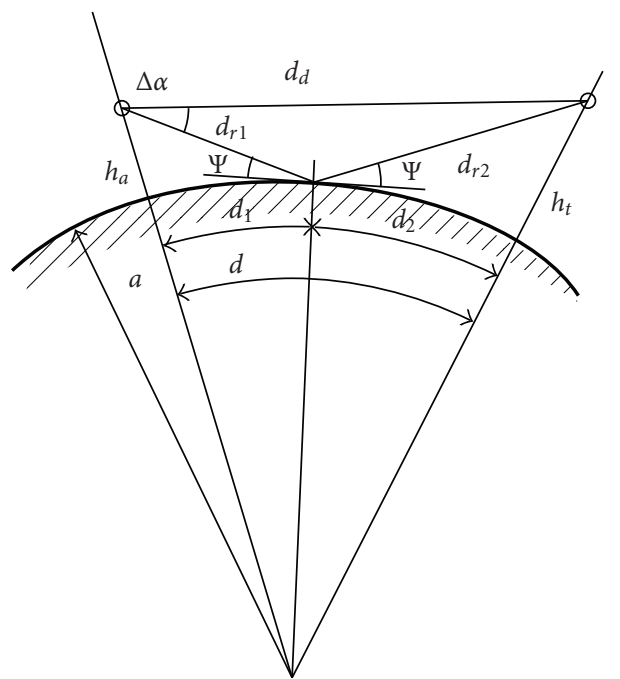

FIGURE 2: Multipath problem for an antenna (left) and a target (right) above a spherical sea surface.

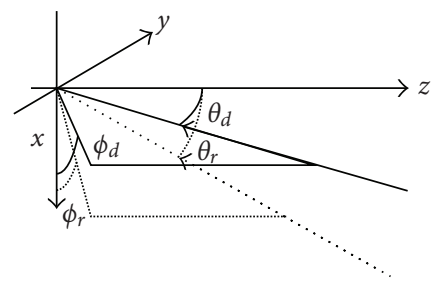

(a)

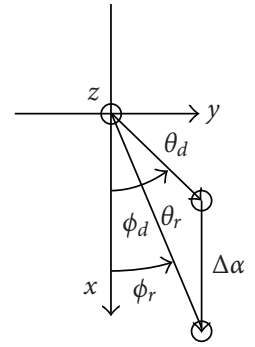

(b)
Figure 3: Antenna reference system 3D view (a) showing the angles characterizing the direction of arrival of the direct and reflected ray. (b) $2 \mathrm{D}$ view of the $x y$ plane, with highlighted the difference in the angle of arrival $\Delta \alpha$.

More accurate models do exist, taking into account earth curvature (Figure 2), and these are used in some papers [5] yet it is easy to show that the differences in $\psi, \Delta \alpha$, and $d_{d}-d_{r}$ are very small for targets low on sea and closer than $5000 \mathrm{~m}$, so the flat-earth model can be safely assumed. In any case, it is easy to show that the effect of the spherical earth is merely a shift in distance of the tracking error and does not affect the error peak values. In both cases, the signal received by the antenna is

$$
E=P\left(\theta_{d}, \phi_{d}\right) \frac{e^{-j k d_{d}}}{d_{d}}+\Gamma_{v, h} D P\left(\theta_{r}, \phi_{r}\right) \frac{e^{-j k d_{r}}}{d_{r}},
$$

$P(\theta, \phi)$ being the pattern of the antenna, $\Gamma_{h, v}$ the appropriate reflection coefficient (horizontal $h$ or vertical $v$ polarization), as discussed here below, and $D$ the spreading factor which is introduced by the spherical earth, if the spherical model is considered. The reference system chosen is a spherical one, with the $z$-axis pointing in the boresight direction of the antenna and the $x$-axis pointing downward. Figure 3 shows this reference. It is important to note that this is fully $3 \mathrm{D}$. 
The difference in the direction of arrival $\Delta \alpha$ is of course vertical since the incident and reflected rays always lie in a vertical plane. It is thus possible to compute the $3 \mathrm{D}$ direction of arrival of the reflected ray once the direction of arrival of the direct ray $\left(\theta_{d}, \phi_{d}\right)$ and $\Delta \alpha$ are known:

$$
\begin{gathered}
\theta_{r}=\cos ^{-1}\left(\cos \theta_{d} \cos \Delta \alpha+\sin \theta_{d} \sin \Delta \alpha \cos \left(\pi-\phi_{d}\right)\right), \\
\phi_{r}=\phi_{d}-\sin ^{-1}\left(\frac{\sin \Delta \alpha \sin \left(\pi-\phi_{d}\right)}{\sin \theta_{r}}\right) .
\end{gathered}
$$

For what concerns the reflection coefficient, the case of a smooth flat sea is trivial:

$$
\begin{gathered}
\Gamma_{h}^{0}=\frac{\sin \psi-\sqrt{n^{2}-\cos ^{2} \psi}}{\sin \psi+\sqrt{n^{2}-\cos ^{2} \psi}}, \\
\Gamma_{v}^{0}=\frac{n^{2} \sin \psi-\sqrt{n^{2}-\cos ^{2} \psi}}{n^{2} \sin \psi+\sqrt{n^{2}-\cos ^{2} \psi}},
\end{gathered}
$$

$n$ being the index of refraction:

$$
n^{2}=\frac{\sigma+j \omega \epsilon}{j \omega \epsilon_{0}} .
$$

For the sea, typical values are $\sigma=4.64 \mathrm{Sm}^{-1}$ and $\epsilon=$ $81 \epsilon_{0}, \epsilon_{0}=8.854 \times 10^{-12} \mathrm{Fm}^{-1}$ being the free space permittivity.

For a rough sea, the electromagnetic model is that presented in [5], which, briefly, distinguishes in the reflection coefficient $\Gamma_{v, h}$ a specular component $\Gamma_{v, h}^{s}$ and a diffused component $\Gamma_{v, h}^{h}$, so that

$$
\Gamma_{v, h}=\Gamma_{v, h}^{s}+\Gamma_{v, h}^{d}
$$

with

$$
\begin{gathered}
\Gamma_{v, h}^{s}=\Gamma_{v, h}^{0} e^{-2\left[2 \pi \sigma_{h} \sin \psi / \lambda\right]^{2}}, \\
\Gamma_{v, h}^{d}=\sqrt{2} \Xi \Gamma_{h, v}^{0} e^{j \bar{\phi}},
\end{gathered}
$$

where $\sigma_{h}$ is the standard deviation of the stochastic process defining the sea surface roughness, $\bar{\phi}$ is a random number with uniform distribution in the $[0,2 \pi]$ range, $\lambda$ is the free space wavelength at the radar working frequency, and $\Xi$ is a heuristic coefficient developed on the basis of a best fit on experimental data:

$$
\Xi=0.77\left(1-e^{-4 \pi\left(\sigma_{h} / \lambda\right) \sin \psi}\right) e^{-4.73\left(\sigma_{h} / \lambda\right) \sin \psi} .
$$

Further details can be found in [5]; here it is worth noticing that effects of the roughness are more relevant for $\sigma_{h}$ comparable with the radar wavelength.

More sophisticated models for sea scattering do exist, as models taking into account Bragg scattering and the other models enumerated in [4] or the two scale model described in [18]. These models are mainly used in remote sensing and polarimetry, and their aim is that to accurately model the backscattering of the sea since that is the main objective

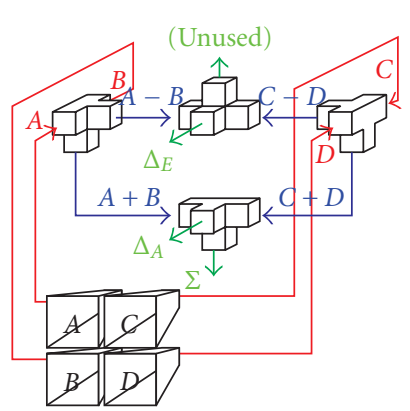

(a)

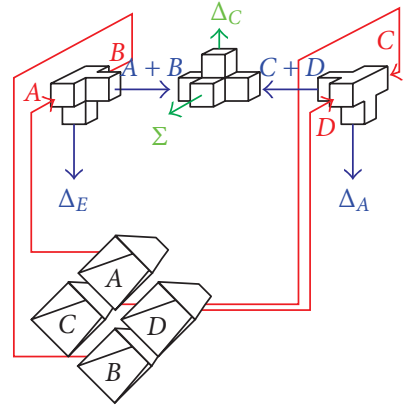

(b)
Figure 4: Comparator networks for the standard monopulse (a) and the crossfeed monopulse (b). In both images primary channels are in red, while first stage elaborations in blue and final outputs in green. The crossfeed setup is simpler not only because it has one less magic-T but also because two outputs are immediately available after the first stage.

there. Here, the sea scattering is rather a noise and is the randomness in phase of the diffuse term, bound to the fine roughness, to have the larger impact in monopulse errors. In this framework, the model in $[5,6]$ for the conventional monopulse is perfectly fit.

\section{Standard and Crossfeed Monopulses}

The monopulse antenna in its standard configuration synthesizes three separate patterns.

(i) A sum $(\Sigma)$ pattern, exhibiting a maximum in the boresight direction;

(ii) An elevation difference pattern $\left(\Delta_{E}\right)$, exhibiting an horizontal line of nulls passing through the boresight direction;

(iii) An azimuth difference pattern $\left(\Delta_{A}\right)$, exhibiting a vertical line of nulls passing through the boresight direction.

These patterns are usually synthesized by creating four different beams, or primary channels named here $A, B, C$, and $D$, by using four separate feeds on a reflector antenna or by subdividing an array into four subarrays. Each beam can be slightly squinted with respect to the boresight direction or simply placed at an offset with respect to the phase center of the whole antenna [1-3].

The primary channels are then combined in a comparator network, which usually comprises four magic- $\mathrm{T}$ junctions in the standard case. For what concerns a standard monopulse, the comparator network (Figure 4) produces the outputs

$$
\begin{gathered}
\Sigma=A+B+C+D, \\
\Delta_{A}=A+B-C-D, \\
\Delta_{E}=A-B+C-D .
\end{gathered}
$$




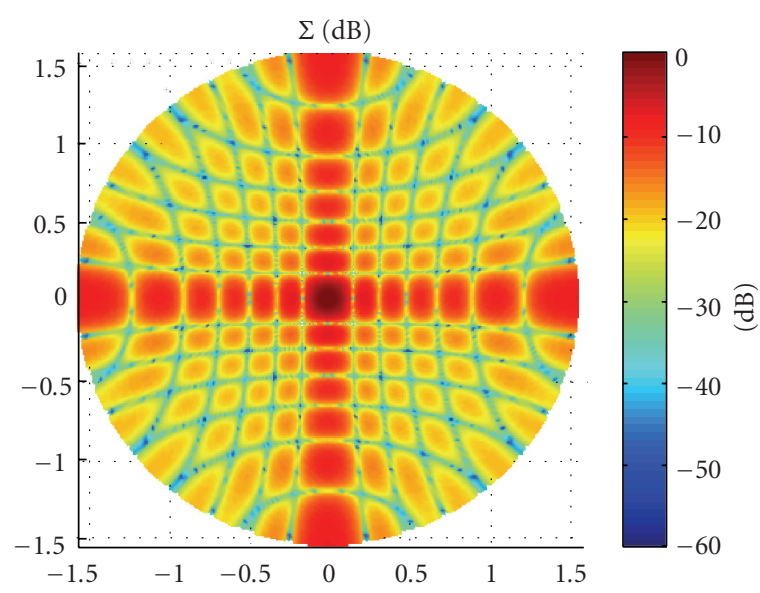

(a)

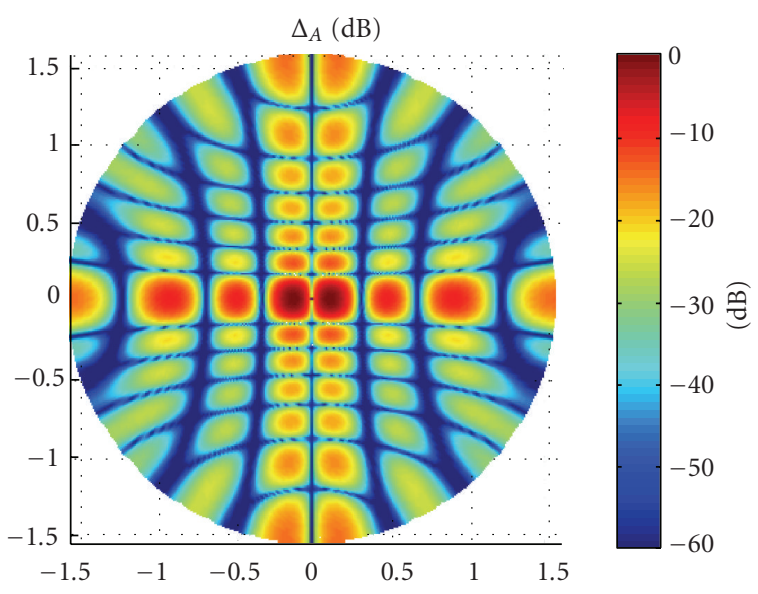

(b)

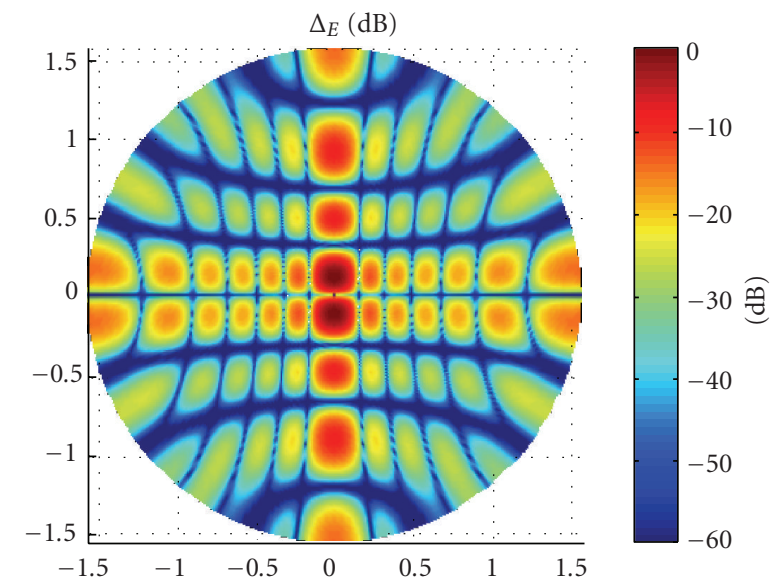

(c)

FIGURE 5: Patterns for a standard monopulse. Patterns are given in a polar reference where the spherical angles $(\theta, \phi)$ are mapped onto $(\rho, \phi)$ coordinates. It is very evident the vertical line of nulls in the azimuthal difference pattern and the horizontal line of nulls in the elevation difference pattern.

The corresponding radiation diagrams are reported in Figure 5 for an ideal $6 \times 6$ array of isotropic sources with a $0.8 \lambda$ spacing.

Tracking is attained by evaluating the error signals in elevation and azimuth:

$$
\epsilon_{E}=\frac{\Delta_{E}^{p}}{\Sigma}, \quad \epsilon_{A}=\frac{\Delta_{A}^{p}}{\Sigma}
$$

and minimizing them. It will be apparent form Section 4 how this approach is severely influenced by multipath.

For what concerns the crossfeed, the primary channels are arranged differently and are combined in a slightly different way, by using just 3 magic-T junctions (Figure 4):

$$
\begin{gathered}
\Delta_{A}=A-B, \\
\Delta_{E}=C-D, \\
\Delta_{C}=A+B-C-D .
\end{gathered}
$$

The synthesized patterns, again for a $6 \times 6$ array of isotropic sources with a $0.8 \lambda$ spacing, are reported in
Figure 6. The elevation error is now corrected by taking into account the quadrature component of the $\Delta_{E}$ channel as well as both the in-phase and quadrature components of the new cross-channel:

$$
\epsilon_{E}=\frac{\Delta_{E}^{p}}{|\Sigma|}-\frac{\Delta_{C}^{p}}{|\Sigma|} \frac{\Delta_{E}^{q}}{\Delta_{C}^{q}} ; \quad \epsilon_{A}=\frac{\Delta_{A}^{p}}{\Sigma} .
$$

This leads theoretically to the complete cancellation of multipath contribution. In subsequent sections, it will be shown how this is strongly dependent on the accuracy and tolerances of the comparator network building the secondary channels from the primary ones, and which is usually manufactured in rectangular waveguide. It will also be shown how it is dependent on the roll and pitch of the ship. An enhanced correction will also be presented.

\section{Ideal Behavior}

As a first assessment of the behavior of the two feed setups an analysis of a system comprising an antenna $10 \mathrm{~m}$ above 


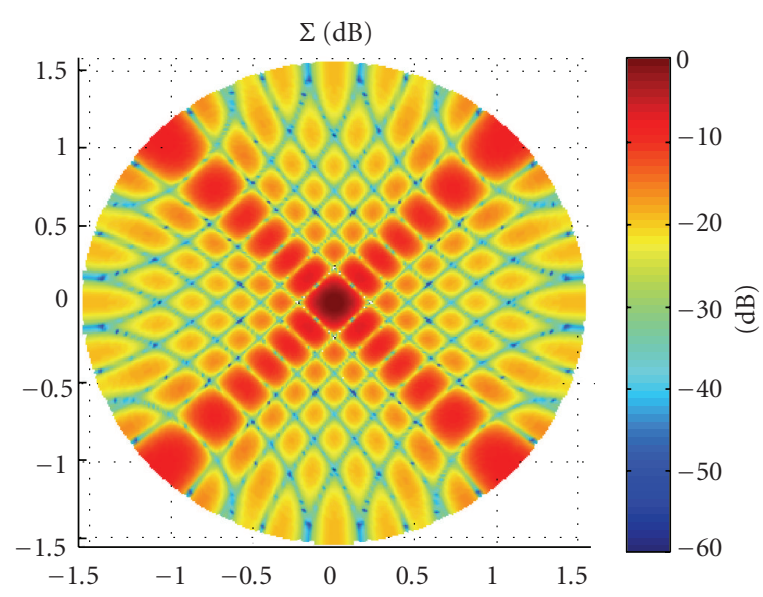

(a)

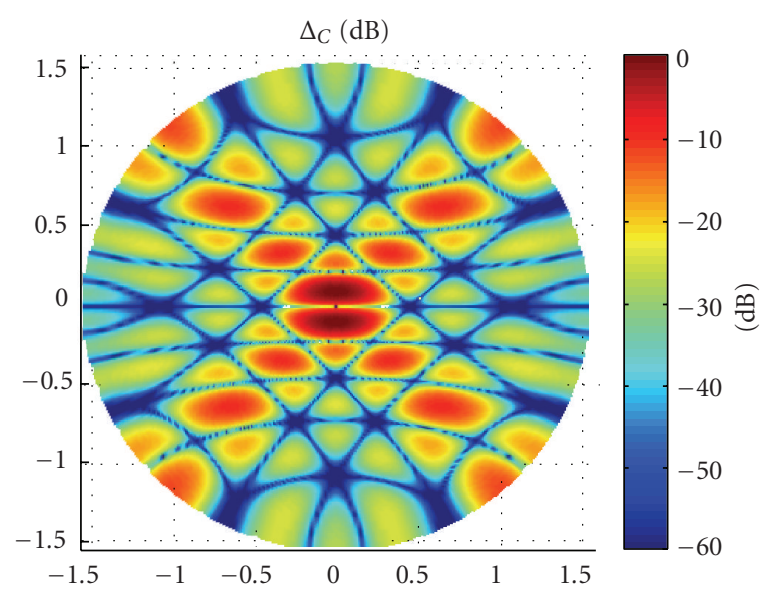

(c)

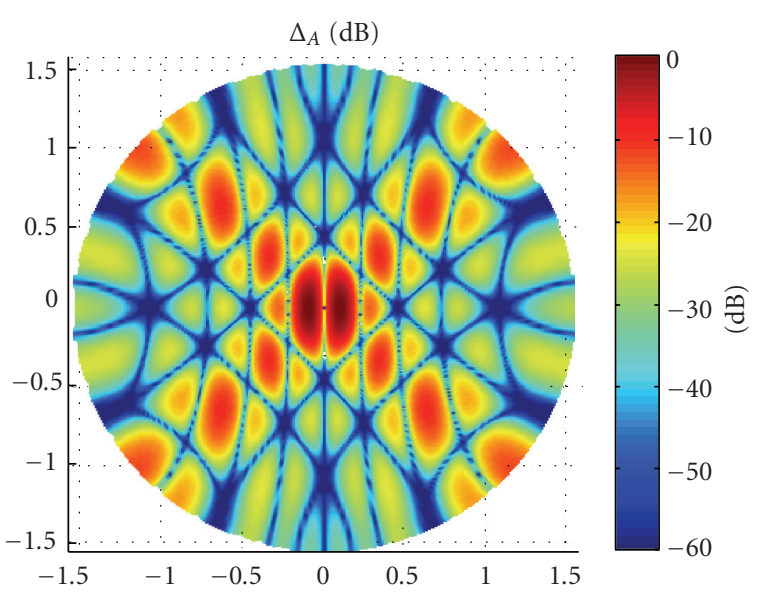

(b)

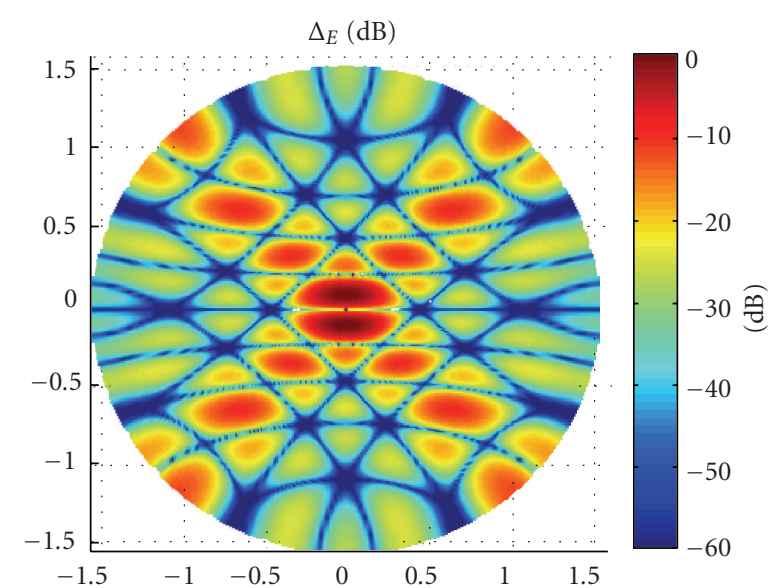

(d)

Figure 6: Patterns crossfeed monopulse. Patterns are given in a polar reference where the spherical angles $(\theta, \phi)$ are mapped onto $(\rho, \phi)$ coordinates. It is very evident the vertical line of nulls in the azimuthal difference pattern and the horizontal line of nulls in the elevation difference pattern. The cross-difference pattern presents two lines of nulls at $\pm 45^{\circ}$.

sea level and a target $20 \mathrm{~m}$ above sea level closing to the radar from $5000 \mathrm{~m}$ down to $100 \mathrm{~m}$ is presented. The standard deviation of the sea roughness is $\sigma_{h}=\lambda / 2$, being $\lambda$ the wavelength of the radar frequency, being this a worst case for (9).

Figure 7 reports the tracking error. This error is defined as the difference, in elevation and azimuth between the antenna pointing direction $\left(\theta_{a}, \phi_{a}\right)$ and the actual target direction $\left(\theta_{d}, \phi_{d}\right)$. For an easier comparison between different antennas these errors are usually normalized with respect to the $3 \mathrm{~dB}$ beamwidth (HPBW) of the antenna:

$$
e_{\theta}=\frac{\theta_{a}-\theta_{d}}{\mathrm{HPBW}} ; \quad e_{\phi}=\frac{\phi_{a}-\phi_{d}}{\mathrm{HPBW}} .
$$

Since in the case at hand the rough sea reflection coefficient is a stochastic process a statistical error is computed and plotted. In Figure 7 the red curve is the average error whereas the two blue curves show the standard deviation limit around the average value. It is apparent how the sea roughness does not degrades the performances significantly.
A similar simulation is performed for the crossfeed monopulse, with the same configuration. No figure is reported for this case since the crossfeed monopulse leads, in the ideal case, to the complete cancellation of the multipath, hence no tracking error is present. Since the cancellation occurs without any explicit knowledge of the reflection coefficient, the presence of the stochastic process does not affect the error, which remains zero also in the presence of sea roughness.

\section{Performances in Presence of Manufacturing Errors}

The case presented in the former section, even if it presents a rough sea, is still ideal inasmuch the radar antenna and its comparator network are ideal. It is interesting, as a first investigation, to verify the effect of a nonideal comparator over the tracking capabilities.

If a relatively large phase error of $5^{\circ}$ is introduced in any of the primary or secondary channels of the standard 


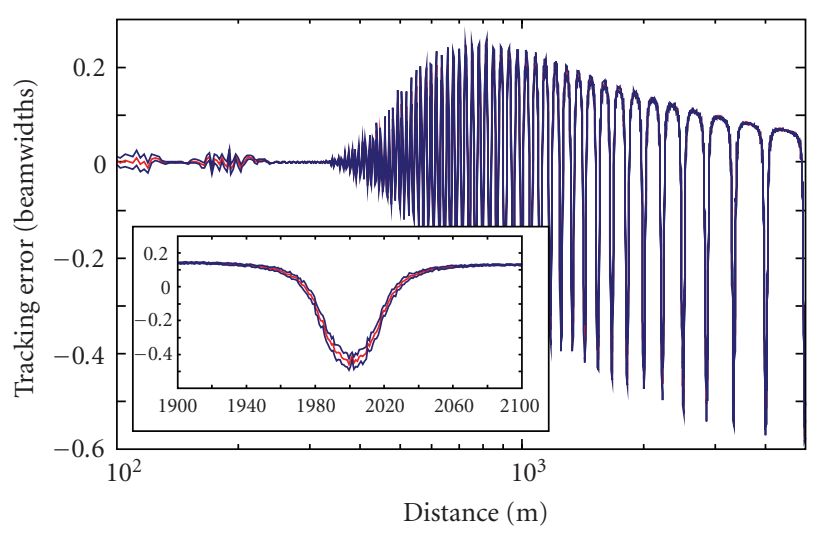

Figure 7: Tracking error, as a function of distance, for the standard monopulse over a rough sea. Red line: average value; blue lines: average plus or minus the standard deviation. In the inset a zoom over a small distance range.

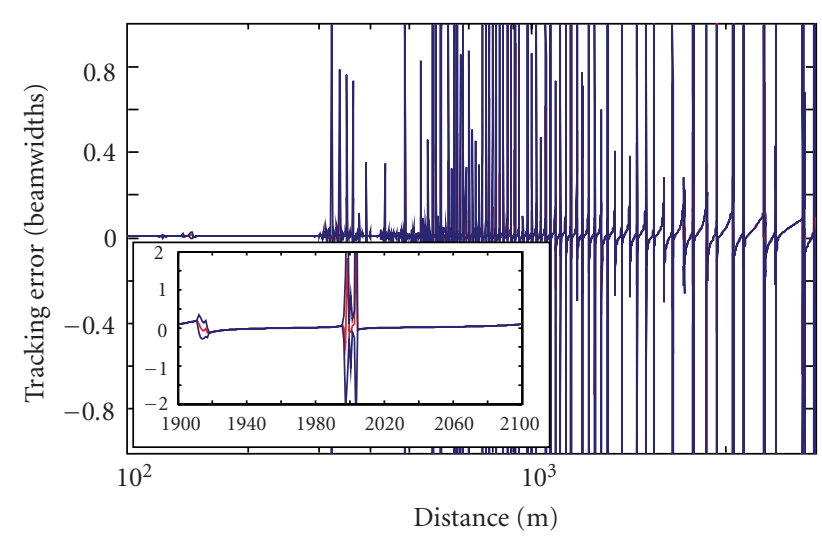

Figure 8: Tracking error, as a function of distance, for the crossfeed monopulse over a rough sea, with a $2^{\circ}$ error on the primary channel A. Red line: average value; blue lines: average plus or minus the standard deviation. In the inset a zoom over a small distance range.

monopulse, no relevant effect is present and the error stays as that of Figure $7.5^{\circ}$ is a relatively large phase error since current manufacturing capabilities for waveguide comparators can achieve accuracies of $1^{\circ}$ or $2^{\circ}$. If, on the other hand, a relatively small $2^{\circ}$ error is introduced in the crossfeed monopulse radar, the effect is very evident and peaks of error on a discrete set of distances arise (Figure 8). This behavior is basically the same whichever channel is affected by the error and presents small range intervals where the average error is very high and, which is more relevant, the standard deviation of the error is extremely high.

The disruptive effect of even very small errors is evident in Figures 9 and 10. In the first figure, the error function for varying elevation angle is presented at two given distances for both the standard and the crossfeed ideal monopulse. It is evident how at $2000 \mathrm{~m}$ the standard monopulse radar behaves worse than at $1950 \mathrm{~m}$, producing in both cases an appreciable error. The crossfeed monopulse, on the other hand, exactly points the target.

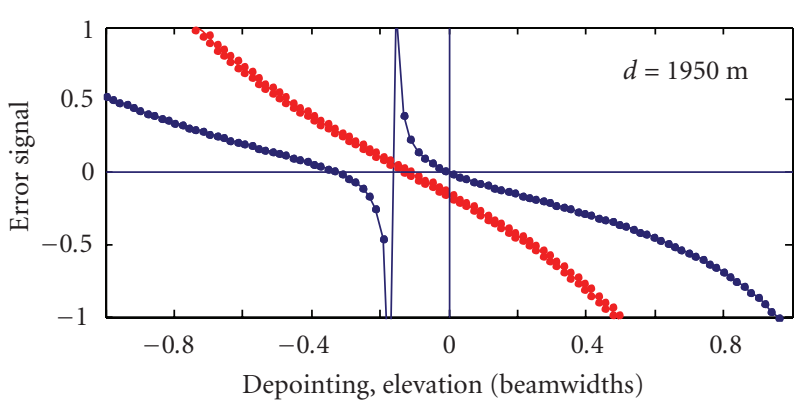

(a)

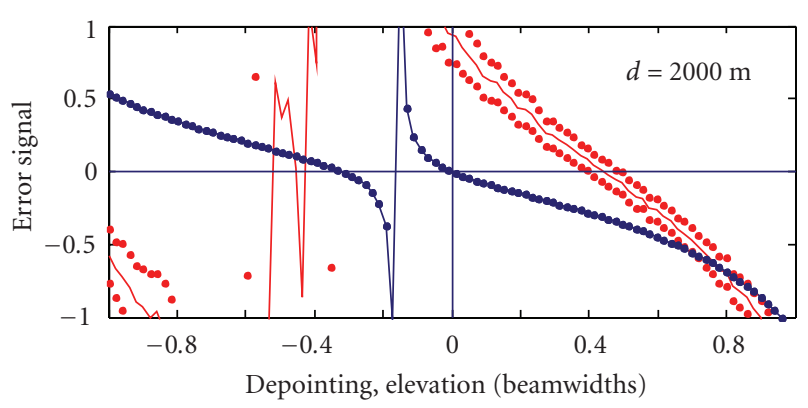

(b)

FIGURE 9: Error function, as a function of elevation error, for the standard and crossfeed monopulse over a rough sea, in case of ideal comparator. (a) Blue: crossfeed; solid line: average; bullets: standard deviation limits around the average values. (b) Red: standard feed; solid line: average; bullets: standard deviation limits around the average values.

Figure 10, on the other hand, shows how a small $1^{\circ}$ error introduced gives practically no effect at $1950 \mathrm{~m}$ and does not significantly degrade the standard monopulse at $2000 \mathrm{~m}$, while the crossfeed monopulse behavior is completely spoiled. This can be ascribed to the presence in (7) of the ratio between quadrature components. The ratio becomes very critical, due to the small value of the denominator, when the difference between the direct and reflected path is an integer number of half wavelengths. Figure 11 indeed shows on the same graph the tracking error of a crossfeed monopulse and the fractional part, with respect to lambda, of the path difference between the direct and reflected rays. It is apparent how error peaks occur where such a path distance is an integer number of wavelengths.

Since the location of the distances at which the crossfeed behavior is critical is a function of wavelength, and hence frequency, it is possible to overcome this limitation by a frequency agility approach. Figure 12 shows the error as a function of distance for the crossfeed monopulse at $f_{0}$ and $1.01 f_{0}$. It is evident how a $1 \%$ variation in the frequency shifts the critical distances significantly.

\section{Performances in Presence of Roll and Pitch}

As a last set of numerical tests, the sensitivity of the monopulse radars to ship's movements, namely, roll and pitch, has been considered. 


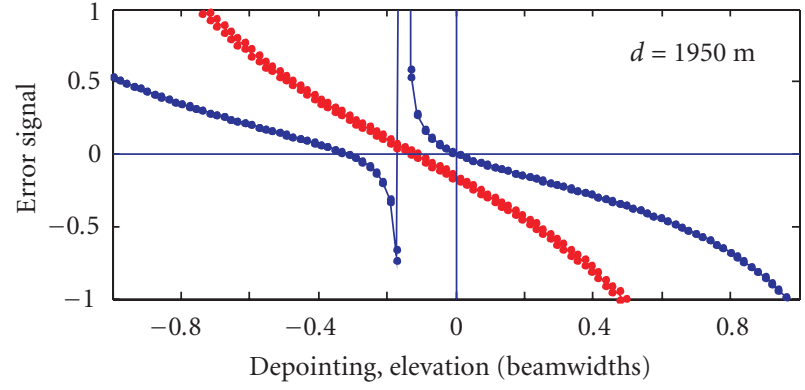

(a)

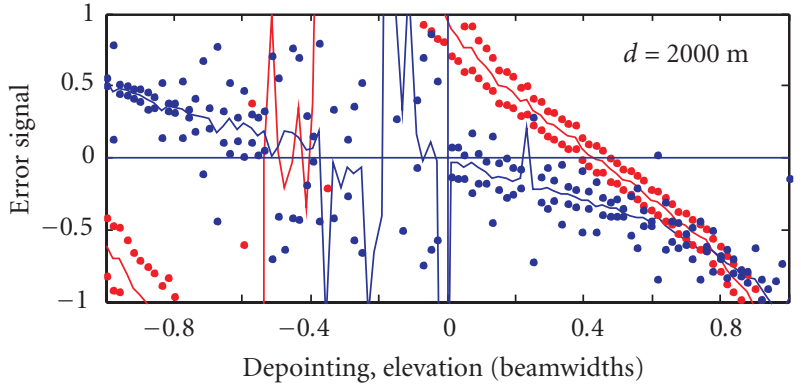

(b)

Figure 10: Error function, as a function of elevation error, for the standard and crossfeed monopulse over a rough sea, in presence of $1^{\circ}$ phase error in one of the primary channel. (a) Blue: crossfeed; solid line: average; bullets: standard deviation limits around the average values. (b) Red: standard feed; solid line: average; bullets: standard deviation limits around the average values.

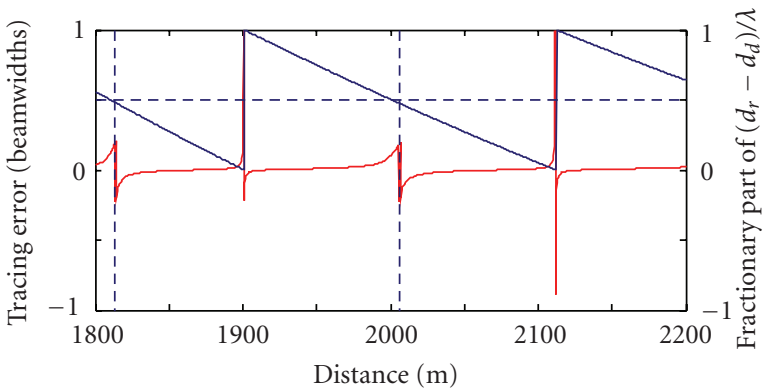

FiguRE 11: Tracking error (red curve) for a crossfeed monopulse as a function of distance. The blue curve shows the differences between the direct and reflected paths as fractions of wavelengths.

The terms roll and pitch refer to standard ship movements along its main horizontal axes. Figure 13 shows roll and pitch movements and their consequences for a target at an azimuth of $90^{\circ}$ with respect to the ship bow. Let us consider the incidence plane, that vertical plane containing both the direct and the sea-reflected ray arriving to the antenna. The incidence plane is vertical with respect to the sea and, in absence of roll and pitch, is also vertical with respect to the ship and antenna reference. In the present case, it is also perpendicular to the ship axis.

When rolls occur, the roll axis is perpendicular with respect to the incidence plane (Figures 13(a) and 13(b)) then the incidence plane stays vertical also in the rotated antenna reference. The roll effect is merely an elevation difference which does not affect multipath. On the other hand, when pitches occur, and the rotation axis is parallel to the incidence plane (Figures 13(c) and 13(d)), then the incidence plane is not vertical any more in the antenna reference. This implies that the sea-reflected signal arrives "sideways" with respect to the antenna and is detected also by the $\Delta_{A}$ channel, hence the multipath also affects the azimuth tracking capabilities. If the target is not at $90^{\circ}$ with respect to the bow, roll and pitch movements interact in a more complex way but it is always possible to reduce them to an elevation difference and in a tilt in the horizontal plane reference in the antenna boresight direction. Only the latter of this needs to be considered and is investigated in this section.
A test case with a $2^{\circ}$ phase error affecting the $\Delta_{E}$ channel and a $10^{\circ}$ pitch have been considered. The pitch consequence is that multipath affects also the azimuth tracking. Figure 14 shows how, with just a $10^{\circ}$ roll, the elevation tracking error basically remains the same, and is entirely due to the phase error in the network. On the other hand, the azimuth error, which is ideally zero if the antenna is not rolled, starts to be affected by the multipath and presents a behavior which is very similar, but on a more limited range, due to the limited roll, to that shown in Figure 7 for the elevation error of a standard monopulse.

To try to overcome this issue a crossfeed correction on the azimuth channel is also suggested:

$$
\epsilon_{E}=\frac{\Delta_{E}^{p}}{|\Sigma|}-\frac{\Delta_{C}^{p}}{|\Sigma|} \frac{\Delta_{E}^{q}}{\Delta_{C}^{q}} ; \quad \epsilon_{A}=\frac{\Delta_{A}^{p}}{|\Sigma|}-\frac{\Delta_{C}^{p}}{|\Sigma|} \frac{\Delta_{A}^{q}}{\mid \Delta_{C}^{q}} .
$$

With these latter equations the crossfeed monopulse behavior is enhanced and the tracking error becomes that in Figure 15. It can be noticed how the error behavior is the same for both elevation and azimuth and that the elevation error is larger. This is due to the limited roll angle. By increasing the roll angle the elevation error decreases and the azimuth error increases up to $45^{\circ}$ roll, where they become the same. 


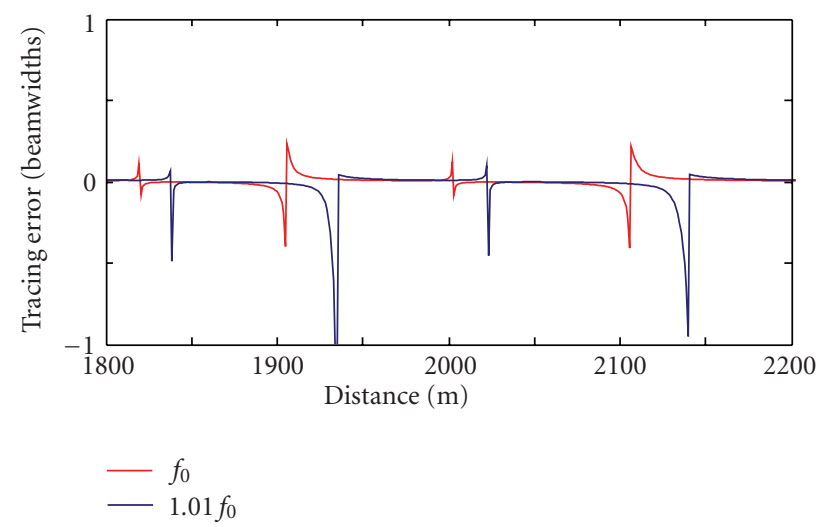

Figure 12: Error function, as a function of elevation error, for two different working frequencies differing by $1 \%$ when the crossfeed comparator is affected by a $2^{\circ}$ error on the Elevation secondary channel.

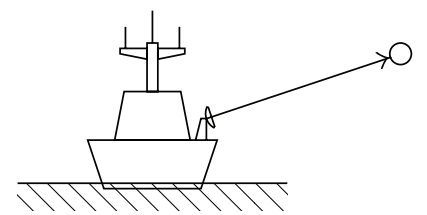

(a)

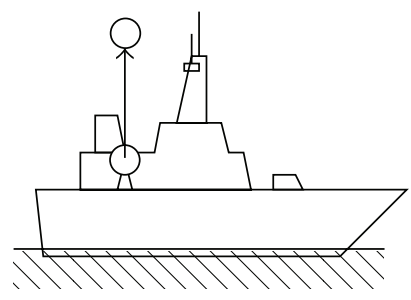

(c)

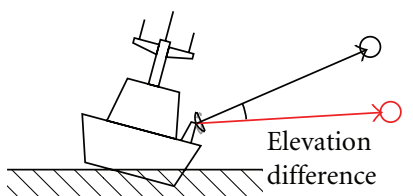

(b)

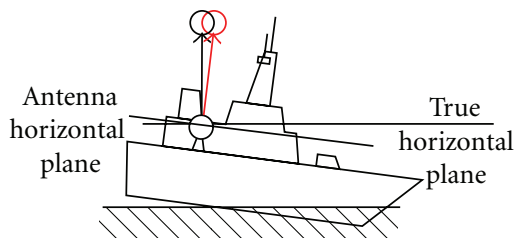

(d)

FIGURE 13: Roll and pitch effects: variation in target elevation $(a, b)$; rotation of the antenna horizontal reference plane (c, d).

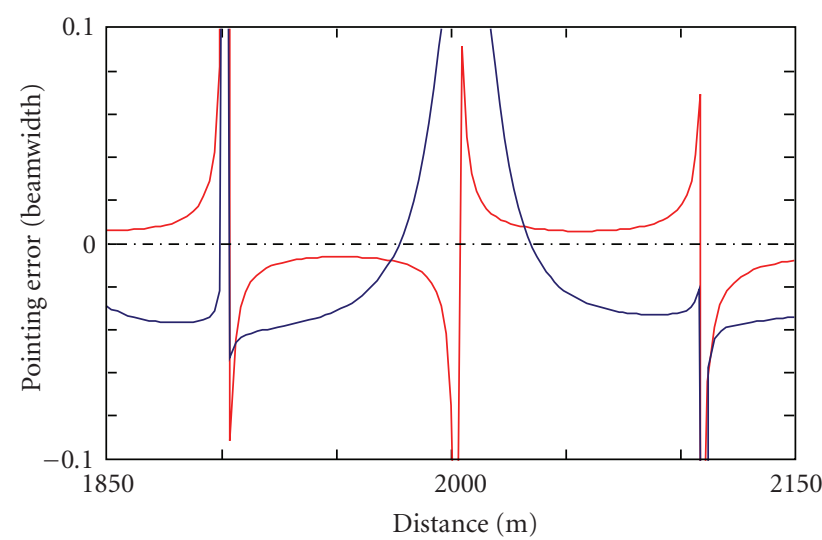

FIGURE 14: Pointing error, in elevation (red) and azimuth (blue) for a crossfeed monopulse with a $10^{\circ}$ inclination around antenna boresight direction (roll or pitch). A $2^{\circ}$ phase error on the elevation secondary channel is also present.

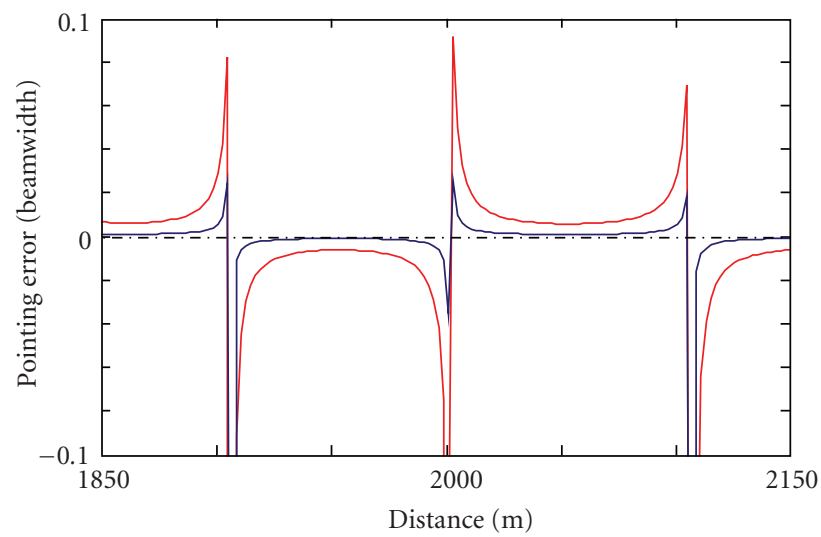

FIGURe 15: Pointing error, in elevation (red) and azimuth (blue) for an enhanced crossfeed monopulse with a $10^{\circ}$ inclination around antenna boresight direction (roll or pitch). A $2^{\circ}$ phase error on the elevation secondary channel is also present. 


\section{Conclusions}

The crossfeed monopulse has been studied and its performances assessed for a marine environment with rough sea scattering multipath. The crossfeed behavior has been studied in presence of small phase errors in the comparator network, showing its sensitivity to these errors and a possible solution by resorting to frequency agility. Finally, the performances have also been studied in presence of antenna roll and pitch, defining a new error with crossfeed correction also on the azimuth.

\section{References}

[1] J. T. Nessmith and W. T. Patton, “Tracking antennas," in Antenna Engineering Handbook, R. C. Johnson and H. Jasik, Eds., McGraw-Hill, New York, NY, USA, 1984.

[2] M. I. Skolnik, Introduction to Radar Systems, McGraw-Hill, New York, NY, USA, 1980.

[3] S. M. Sherman, Monopulse Principles and Techniques, Artech House, Norwood, MA, USA, 1984.

[4] G. R. Valenzuela, "Theories for the interaction of electromagnetic and oceanic waves-a review," Boundary-Layer Meteorology, vol. 13, no. 1-4, pp. 61-85, 1978.

[5] T. Griesser and C. A. Balanis, "Oceanic low-angle monopulse radar tracking errors," IEEE Journal of Oceanic Engineering, vol. 12, no. 1, pp. 289-295, 1987.

[6] C. A. Balanis, R. Hartenstein, and D. DeCarlo, "Multipath interference for in-flight antenna measurements," IEEE Transactions on Antennas and Propagation, vol. 32, no. 1, pp. 100104, 1984.

[7] W. D. Blair and M. Brandt-Pearce, "Statistics of monopulse measurements of Rayleigh targets in the presence of specular and diffuse multipath," in Proceedings of IEEE Radar Conference, pp. 369-375, May 2001.

[8] W. D. Blair and M. Brandt-Pearce, "Statistics of monopulse measurements for tracking targets in the presence of seasurface induced multipath," in Proceedings of IEEE Aerospace Conference, pp. 49-58, March 1998.

[9] A. R. Miller, R. M. Brown, and E. Vegh, "New derivation for the rough surface reflection coefficient and for distribution of sea-wave elevations," IEE Proceedings $H$, vol. 131, no. 2, pp. 114-116, 1984.

[10] A. R. Miller and E. Vegh, "A family of curves for the rough surface reflection coefficient," Tech. Rep. 8898, Naval Research Lab, Washington, DC, USA, 1985.

[11] A. S. P. Beckmann, The scattering of elettro-magnetic wave from rough surface, Artech House, Norwood, Mass, USA, 1987.

[12] I. D. Macey, "Measurements of SSR bearing errors due to site obstructions," in Proceedings of the Radar 92 International Conference, pp. 137-142, October 1992.

[13] A. J. McDevitt, "A tracker for monopulse ssr," in Proceedings of IEE Colloquium on State Estimation in Aerospace and Tracking Applications, pp. 8/1-8/3, December 1989.

[14] E. Bossé, R. M. Turner, and E. S. Riseborough, "Modelbased multifrequency array signal processing for low-angle tracking," IEEE Transactions on Aerospace and Electronic Systems, vol. 31, no. 1, pp. 194-210, 1995.

[15] A. E. Schenkel, "Cross-feed monopulse-a specific method to eliminate mistracking over sea," in IEE Radar-87, October 1987.

[16] G. Giunta, L. Lucci, G. Pelosi, C. Riminesi, S. Selleri, and F. Serrano, "Analysis of monopulse crossfeed performances in presence of rough sea multipath," in Proceedings of the Mediterranean Microwave Symposium, pp. 343-346, September 2006.

[17] G. Giunta, L. Lucci, G. Pelosi, C. Riminesi, S. Selleri, and F. Serrano, "Tolerance manufacturing effects in crossfeed monopulse radars in presence of rough sea scattering," in Proceeding of IEEE Antennas and Propagation Society International Symposium, pp. 4817-4820, Honolulu, Hawaii, USA, June 2007.

[18] S. H. Yueh, "Modeling of wind direction signals in polarimetric sea surface brightness temperatures," IEEE Transactions on Geoscience and Remote Sensing, vol. 35, no. 6, pp. 1400-1418, 1997. 

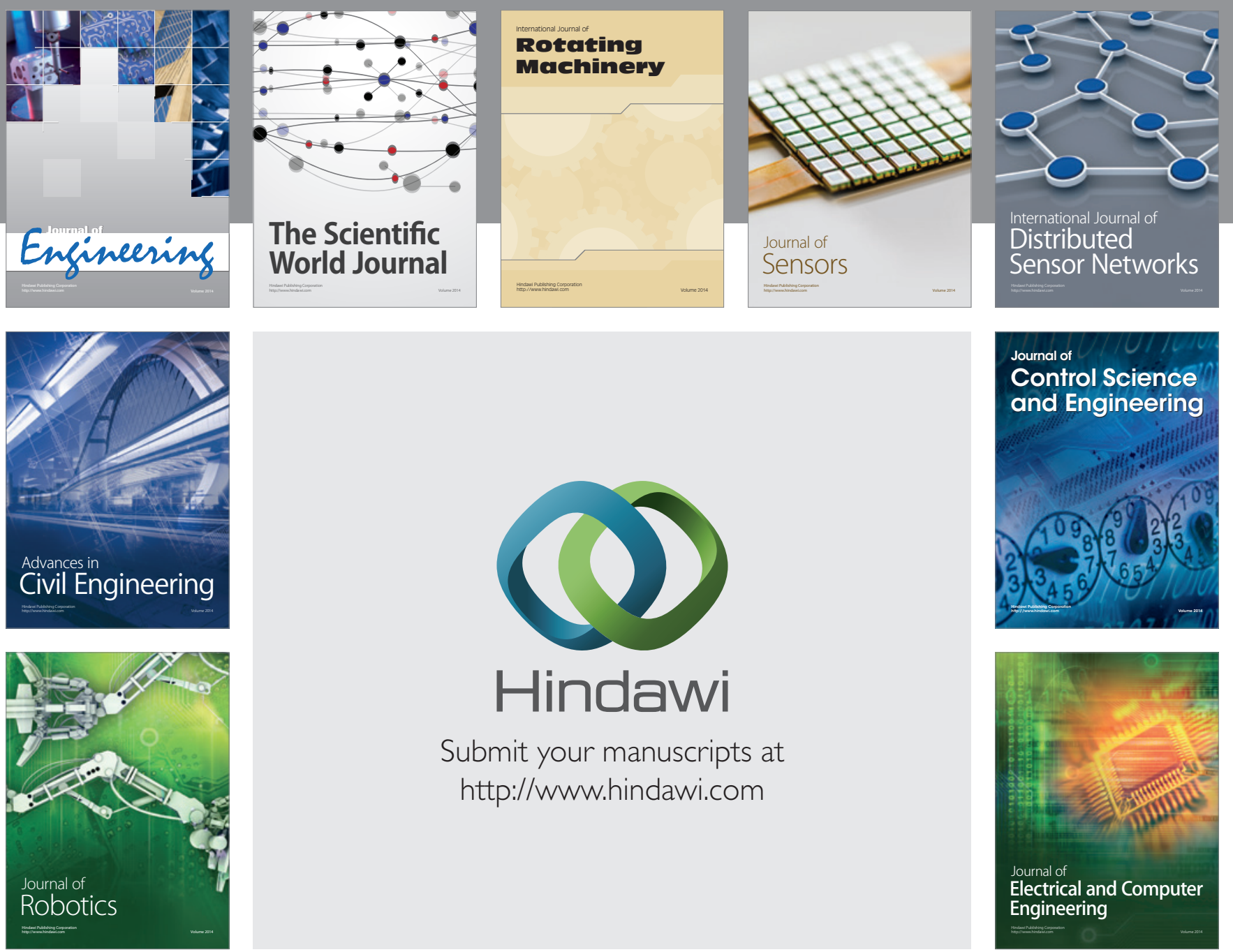

Submit your manuscripts at

http://www.hindawi.com
